\title{
On power stable quasi-copulas
}

\author{
Anna Kolesárová ${ }^{1}$ Radko Mesiar ${ }^{2}$ \\ ${ }^{1}$ Institute of Information Engineering, Automation and Mathematics, \\ Faculty of Chemical and Food Technology, Slovak University of Technology in Bratislava, \\ Radlinského 9, 81237 Bratislava 1, Slovakia \\ e-mail: anna.kolesarova@stuba.sk \\ ${ }^{2}$ Department of Mathematics and Descriptive Geometry, \\ Faculty of Civil Engineering, Slovak University of Technology in Bratislava, \\ Radlinského 11, 81368 Bratislava 1, Slovakia \\ e-mail: radko.mesiar@stuba.sk \\ and \\ Centre of Excellence IT4Innovations, \\ Division University of Ostrava, IRAFM, \\ Czech Republic
}

\begin{abstract}
In our contribution we discuss the power stability of 1-Lipschitz binary aggregation functions. The main result is the characterization of power stable quasi-copulas by means of their dependence functions. The notions are illustrated by examples.
\end{abstract}

Keywords: Aggregation function, Copula, Power stability, Quasi-Copula

\section{Introduction}

Power stable aggregation functions were investigated and characterized in paper [7] containing a general characterization of continuous power stable binary aggregation functions, characterizations of continuous power stable binary aggregation functions with some additional properties, e.g., with neutral element or annihilator, and also characterization of continuous power stable binary aggregation functions which are associative. In this paper we will study 1-Lipschitz power stable binary aggregation functions. We focus our attention on the class of binary quasi-copulas (quasi-copulas for short). Quasi-copulas can be defined in different ways. Primarily, they were introduced as generalizations of copulas based on the coincidence of values on tracks [1]. Note that a copula is an aggregation function $C:[0,1]^{2} \rightarrow[0,1]$ with neutral element $e=1$ satisfying the supermodular property, i.e., for all $\mathbf{x}, \mathbf{y} \in[0,1]^{2}$,

$$
C(\mathbf{x} \wedge \mathbf{y})+C(\mathbf{x} \vee \mathbf{y}) \geq C(\mathbf{x})+C(\mathbf{y}) .
$$

Quasi-copulas were also shown to be 1-Lipschitz aggregation functions with neutral element $e=1$ [4]. It is also known that the class of all quasicopulas is the lattice closure of the class of all copulas. Even more, for each quasi-copula $Q$ there exist systems of copulas $\left(C_{i}\right)_{i \in I}$ and $\left(D_{j}\right)_{j \in J}$, such that
$Q=\sup _{i \in I} C_{i}=\inf _{j \in J} D_{j}$ (the standard partial ordering of 2-dimensional real functions is considered).

Characterization of power stable copulas which are also known as Extreme Value copulas (EVcopulas for short), is well known. For an exhaustive overview of EV-copulas we refer to [6]. But no attempt to characterize power stable quasi-copulas, or more generally 1-Lipschitz power stable aggregation functions, has been made up to now.

The paper is organized as follows. In the next section, we introduce basic notions and results concerning power stable aggregation functions. Section 3 is devoted to the study of 1-Lipschitz power stable aggregation functions, and especially power stable quasi-copulas. Finally, some concluding remarks are added.

\section{Power stable aggregation functions}

We start with recalling several definitions.

A function $A:[0,1]^{2} \rightarrow[0,1]$ is called an aggregation function if it is monotone (i.e., increasing in each variable) and satisfies the boundary conditions $A(0,0)=0$ and $A(1,1)=1$.

As we are working in the framework of aggregation functions, we will use the following basic definition of quasi-copulas.

A quasi-copula is an aggregation function $Q:[0,1]^{2} \rightarrow[0,1]$ with neutral element $e=1$ that is 1-Lipschitz wrt. $L_{1}$-norm, i.e., for all $\mathbf{x}=\left(x_{1}, y_{1}\right), \mathbf{y}=\left(x_{2}, y_{2}\right)$ in $[0,1]^{2}$,

$$
|Q(\mathbf{x})-Q(\mathbf{y})| \leq|| \mathbf{x}-\mathbf{y} \|_{1}=\left|x_{1}-x_{2}\right|+\left|y_{1}-y_{2}\right| \text {. }
$$

Definition 1 An aggregation function $A:[0,1]^{2} \rightarrow$ $[0,1]$ is called power stable whenever for any constant $r \in] 0, \infty\left[\right.$ and all $(x, y) \in[0,1]^{2}$ it holds

$$
A\left(x^{r}, y^{r}\right)=(A(x, y))^{r}
$$

Continuous power stable binary aggregation functions have been characterized in our recent paper 
[7]. The paper has not been published yet, therefore we recall the following two results from [7], important for our next investigation.

Proposition 1 A continuous function $A:[0,1]^{2} \rightarrow$ $[0,1]$ is a power stable aggregation function if and only if there is a continuous non-zero function $d:[0,1] \rightarrow[0, \infty[$ such that

(i) the function $\left.\left.d_{+}:\right] 0,1\right] \rightarrow\left[0, \infty\left[, d_{+}(t)=\frac{d(t)}{t}\right.\right.$, is decreasing, and $d(0)>0$ or $\lim _{t \rightarrow 0^{+}} d_{+}(t)<\infty$,

(ii) the function $d_{-}:\left[0,1\left[\rightarrow\left[0, \infty\left[, d_{-}(t)=\frac{d(t)}{1-t}\right.\right.\right.\right.$, is increasing, and $d(1)>0$ or $\lim _{t \rightarrow 1^{-}} d_{-}(t)<\infty$,

and for all $(x, y) \in] 0,1\left[^{2}\right.$ it holds

$$
A(x, y)=(x y)^{d\left(\frac{\log x}{\log x y}\right)} .
$$

Functions $d$ characterized in Proposition 1 are called dependence functions, and the class of all such functions $d$ will be denoted by $\mathcal{D}$.

Note that each power stable aggregation function $A$ is continuous on $] 0,1\left[{ }^{2}\right.$. Due to power stability, $\left.A\right|_{0,1\left[^{2}\right.} \equiv 0$ or $\left.A\right|_{0,1\left[^{2}\right.} \equiv 1$, or $A$ is on $] 0,1\left[^{2}\right.$ generated by a function $d$ via (2). If the function $d$ satisfies all conditions given in Proposition 1, then there exists a continuous power stable aggregation function $B:[0,1]^{2} \rightarrow[0,1]$ such that $\left.B\right|_{] 0,1\left[{ }^{2}\right.}=\left.A\right|_{] 0,1[2}$. For more details we refer to [7].

Proposition 2 A continuous power stable aggregation function $A$ generated by a dependence function $d \in \mathcal{D}$ has neutral element $e=1$ if and only if $d(0)=d(1)=1$.

Copulas are particular 1-Lipschitz aggregation functions. EV-copulas were characterized in $[2,9]$ as follows.

Proposition $3 A$ copula $C:[0,1]^{2} \rightarrow[0,1]$ is power stable if and only if there is a convex function $d:[0,1] \rightarrow[0,1]$ such that for all $t \in[0,1]$,

$$
\max \{t, 1-t\} \leq d(t) \leq 1,
$$

and for all $(x, y) \in] 0,1\left[^{2}\right.$,

$$
C(x, y)=(x y)^{d\left(\frac{\log x}{\log x y}\right)}
$$

The dependence functions connected with EVcopulas are the Pickands dependence functions, and the class of all Pickands' dependence functions will be denoted by $\mathcal{P}$.

\section{1-Lipschitz power stable aggregation functions}

It can be checked that for any continuous power stable aggregation function $A:[0,1]^{2} \rightarrow[0,1]$, the partial functions $A(0,),. A(., 0), A(1,$.$) and A(., 1)$ are either constants 0 or 1 , or they are power functions $t^{\alpha}$ with a positive $\alpha$. If $A$ is considered to be 1 Lipschitz, then for the above partial functions there are only the next possibilities: $A(0,),. A(., 0) \in$ $\left\{0,\left.i d\right|_{[0,1]}\right\}$ and $A(1,),. A(., 1) \in\left\{1,\left.i d\right|_{[0,1]}\right\}$. Thus, using the monotonicity of aggregation functions, we can divide all 1-Lipschitz power stable aggregation functions into the next four classes. Namely, the class of aggregation functions for which

(i) $A(0, t)=A(t, 0)=0$ (and then $A(1, t)=$ $A(t, 1)=t)$, i.e., 1-Lipschitz power stable aggregation functions with neutral element $e=1$, which means that the first class is formed by power stable quasi-copulas.

(ii) $A(0, t)=A(1, t)=t, t \in[0,1]$, which gives $A(x, y)=y$, i.e., the second class contains the projection to the second coordinate only, $A=$ $P_{L}$

(iii) $A(t, 0)=A(t, 1)=t, t \in[0,1]$, which gives $A(x, y)=x$, i.e., the third class only contains the projection to the first coordinate only, $A=$ $P_{F}$.

(iv) $A(0, t)=A(t, 0)=t$ (and then $A(1, t)=$ $A(t, 1)=1), t \in[0,1]$. Then $A(t, t)=t^{\alpha}$, $\alpha>0$ and due to the 1-Lipschitz property of $A$, for each $t \in[0,1]$ it holds

$$
A(1, t)-A(t, t)=1-t^{\alpha} \leq 1-t,
$$

i.e., $\alpha \in] 0,1]$. On the other hand, for each $t \in$ $[0,1]$ it has to hold

$$
A(t, t)-A(t, 0)=t^{\alpha}-t \leq t,
$$

which implies $\alpha=1$, and thus $A(t, t)=t$. Consequently, $A(x, y)=\max \{x, y\}$.

The previous discussion shows that the only nontrivial case which has to be clarified, is case (i) concerning power stable quasi-copulas.

Recall that each quasi copula $Q:[0,1]^{2} \rightarrow[0,1]$ is a conjunctive aggregation function, i.e., for all $(x, y) \in[0,1]^{2}, Q(x, y) \leq \min \{x, y\}$. Continuous conjunctive power stable aggregation function can be characterized by means of their dependence functions as follows.

Proposition 4 A continuous power stable aggregation function $A$ generated by a dependence function $d$ is conjunctive if and only if for each $t \in[0,1]$,

$$
d(t) \geq \max \{t, 1-t\} .
$$

Proof.

As mentioned above, $A$ is conjunctive aggregation function if and only if for all $(x, y) \in$ $[0,1]^{2}, A(x, y) \leq \operatorname{Min}(x, y)$.

Observe that the inequality $A(x, y) \leq x$ is for each $(x, y) \in] 0,1[2$ equivalent to

$$
\exp (\log x y) d\left(\frac{\log x}{\log x y}\right) \leq \exp \log x
$$


and next to

$$
d\left(\frac{\log x}{\log x y}\right) \geq \frac{\log x}{\log x y},
$$

which can be written as $d(t) \geq t, t \in[0,1]$.

Similarly, the inequality $A(x, y) \leq y$ is equivalent to $d(t) \geq 1-t$.

Before giving a complete characterization of power stable quasi-copulas, recall that a real function $\varphi:[a, b] \rightarrow \mathbb{R}$ is convex at a point $t_{0} \in[a, b]$, if for each $t \in[a, b]$ and $c \in[0,1]$ it holds

$$
f\left((1-c) t_{0}+c t\right) \leq(1-c) f\left(t_{0}\right)+c f(t) .
$$

The function $\varphi$ is convex on $[a, b]$ if and only if it is convex at each $t_{0} \in[a, b]$.

In the next characterization we will use the convexity of a dependence function $d \in \mathcal{D}$ at the points 0 and 1, i.e., the properties

$$
d(c t) \leq(1-c) d(0)+c d(t),
$$

and

$$
d(c t+1-c) \leq(1-c) d(1)+c d(t)
$$

respectively, valid for all $t \in[0,1]$ and $c \in[0,1]$.

Theorem 1 A continuous function $Q:[0,1]^{2} \rightarrow$ $[0,1]$ is a power stable quasi-copula if and only if it can be written in the form (2) for some dependence function $d \in \mathcal{D}$ such that $d(0)=d(1)=1$, and $d$ is convex at points 0 and 1 .

Proof.

Sufficiency. First observe that for a dependence function $d \in \mathcal{D}$ such that $d(0)=d(1)=1$, the corresponding power stable aggregation function is conjunctive and thus, by Proposition 4 , for all $t \in[0,1]$, $\max \{t, 1-t\} \leq d(t)$. Next, for each $u \in] 0,1[$, consider the function $d_{u}:[0,1] \rightarrow[0,1]$, defined by

$$
d_{u}(t)= \begin{cases}1-\frac{t}{u}+\frac{t}{u} d(u) & \text { if } t \leq u \\ \frac{1-t}{1-u} d(u)+\frac{t-u}{1-u} & \text { otherwise. }\end{cases}
$$

Due to (5) and (6), $d_{u}$ is a convex function satisfying all requirements for Pickands' dependence functions, i.e., $d_{u} \in \mathcal{P}$, and thus it generates via (4) a copula $C_{u}$. Moreover, $d=\inf _{u \in] 0,1[} d_{u}$ and thus, $Q=\sup _{u \in] 0,1[} C_{u}$, i.e., $Q$ is a quasi-copula.

Necessity. Observe that the property $d(0)=d(1)=$ 1 follows from Proposition 2. Suppose that $d$ is not 0 -convex, i.e., there are $t, c \in] 0,1[$ such that $d(c t)>$ $1-c+c d(t)$. From the representation formula (2) we can see that for any $x, y \in\left[0,1\left[\right.\right.$ such that $\frac{\log x}{\log x y}=t$, i.e., $y=x^{(1-t) / t}$, it holds

$$
\begin{aligned}
Q(x, y) & =Q\left(x, x^{(1-t) / t}\right)=\left(x x^{(1-t) / t}\right)^{d(t)} \\
& =x^{d(t) / t}
\end{aligned}
$$

Similarly, $Q\left(x, x^{(1-c t) / c t}\right)=x^{d(c t) / c t}$.

The 1-Lipschitz property of $Q$ ensures that for all $x \in] 0,1[$,

$$
\begin{aligned}
& Q\left(x, x^{(1-t) / t}\right)-Q\left(x, x^{(1-c t) / c t}\right) \\
= & x^{d(t) / t}-x^{d(c t) / c t} \leq x^{(1-t) / t}-x^{(1-c t) / c t} .
\end{aligned}
$$

Next, the continuity of $Q$ implies that the function $f:] 0,1] \rightarrow \mathbb{R}$

$$
f(x)=x^{d(t) / t}-x^{d(c t) / c t}-x^{(1-t) / t}+x^{(1-c t) / c t}
$$

has for each $x \in] 0,1]$ a non-positive value. On the other hand, it holds $f(1)=0$ and

$$
\begin{aligned}
f^{\prime}(1) & =\frac{d(t)}{t}-\frac{d(c t)}{c t}-\frac{1-t}{t}+\frac{1-c t}{c t} \\
& <\frac{d(t)}{t}-\frac{1-c+c d(t)}{c t}-\frac{1}{t}+\frac{1}{c t}=0,
\end{aligned}
$$

i.e., $f$ is strictly decreasing on some neighbourhood of the point 1 . However, then necessarily $f(x)>0$ for some $x$ close to 1 , which is a contradiction.

Example 1 Consider the Pickands dependence functions $d_{1}, d_{2} \in \mathcal{P}$, given by

$$
d_{1}(t)=\max \left\{1-t, \frac{1+t}{2}\right\}, \quad d_{2}(t)=d_{1}(1-t) .
$$

Then $d=\min \left\{d_{1}, d_{2}\right\} \in \mathcal{D}$ generates a proper power stable quasi-copula $Q$ (i.e., $Q$ is not a copula) given by $Q(x, y)=\max \{C(x, y), C(y, x)\}$, where $C$ is the $E V$-copula generated by $d_{1}, C(x, y)=\min \{y, x \sqrt{y}\}$. To see that $Q$ is not a copula, it is enough to consider the points $\mathbf{x}=\left(\frac{1}{4}, \frac{1}{2}\right)$ and $\mathbf{y}=\left(\frac{1}{2}, \frac{1}{4}\right)$. Then

$$
\begin{aligned}
& Q(\mathbf{x} \vee \mathbf{y})+Q(\mathbf{x} \wedge \mathbf{y})=Q\left(\frac{1}{2}, \frac{1}{2}\right)+Q\left(\frac{1}{4}, \frac{1}{4}\right) \\
= & \frac{2 \sqrt{2}+1}{8}<\frac{1}{2}=Q(\mathbf{x})+Q(\mathbf{y}),
\end{aligned}
$$

which means that $Q$ is not supermodular.

The next example shows the importance of the convexity properties of $d$.

Example 2 Consider the function d defined by

$$
d(t)= \begin{cases}1-t & t \in[0,1 / 3] \\ 1 / 3+t & t \in] 1 / 3,1 / 2] \\ 1-t / 3 & t \in] 1 / 2,3 / 4] \\ t & t \in] 3 / 4,1]\end{cases}
$$

see Fig. 1. It is easy to see that $d \in \mathcal{D}, d(0)=$ $d(1)=1$, and that $d$ is 0-convex, but not 1-convex (e.g., for $x=5 / 12$ and $c=6 / 7$ the condition (6) is not satisfied).

If we construct the aggregation function A using (2), we obtain

$$
A(x, y)= \begin{cases}y & x \in] 0,1\left[, 0<y \leq x^{2}\right. \\ \left(x^{4} y\right)^{1 / 3} & x \in] 0,1\left[, x^{2}<y \leq x\right. \\ x^{2 / 3} y & x \in] 0,1[, \quad x<y \leq \sqrt[3]{x} \\ x & x \in] 0,1[, \sqrt[3]{x}<y<1\end{cases}
$$




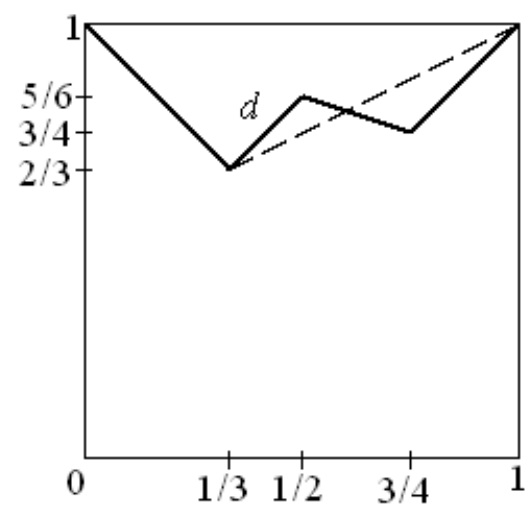

Figure 1: The dependence function $d$ from Example 2 that is not convex at point 1 .

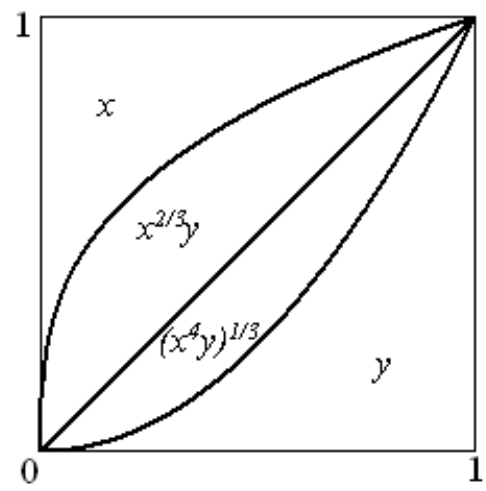

Figure 2: The aggregation function $A$ generated by the dependence function $d$ from Example 2 .

see Fig. 2.

$A$ is 1-Lipschitz in the second variable, i.e., $A(x,$. is 1-Lipschitz for each $x$, but $A$ is not 1-Lipschitz in the first variable. Indeed, if we choose the points $\mathbf{u}=(0.9,0.81)$ and $\mathbf{v}=(0.81,0.81)$, then $A(0.9,0.81)=0.81, A(0.81,0.81)=\sqrt[3]{0.9^{10}}=$ 0.703842 , and thus,

$$
\begin{aligned}
A(0.9,0.81)-A(0.81,0.81) & =0.106158 \\
& >0.09=0.9-0.81,
\end{aligned}
$$

which confirms that $A$ is not 1-Lipschitz in the first variable.

\section{Concluding remarks}

We have clarified the structure of the class of 1Lipschitz power stable aggregation functions, in- cluding the prominent subclass of power stable quasi-copulas. As the main result we have shown the relationship of power stable quasi-copulas and dependence functions convex at points 0 and 1 , satisfying the property $d(0)=d(1)=1$. For more details and some other related results see [8]. In our further investigation, we open the problem of characterization of 1-Lipschitz power stable aggregation functions of higher dimensions.

Acknowledgement The authors kindly acknowledge the support of the grant VEGA 1/0419/13 and the project of Science and Technology Assistance Agency under the contract No. APVV0073-10. Moreover, the work of the second author on this paper has been done in connection with project IT4Innovations Centre of Excellence, reg. no. CZ.1.05/1.1.00/ 02.0070 supported by Research and Development for Innovations Operational Programme financed by Structural Founds of Europe Union and from the means of state budget of the Czech Republic.

\section{References}

[1] C. Alsina, R.B. Nelsen, B. Schweizer, On the characterization of a class of binary operations on distributions functions. Stat. Probab. Lett. 17 (1993) 85-89.

[2] J. Beirlant, Y. Goegebeur, J. Segers, and J. Teugels, Statistics of Extremes: Theory and Applications. John Wiley \& Sons Ltd., Chichester, 2004.

[3] G. Beliakov, A. Pradera, and T. Calvo, Aggregation Functions: A Guide for Practitioners. Springer, Heidelberg, Berlin, New York, 2007.

[4] C. Genest, J.J. Quesada Molina, J.A. Rodríguez-Lallena, and C. Sempi, A characterization of quasi-copulas. J. Multivariate Anal. 69 (1999) 193-205.

[5] M. Grabisch, J.-L. Marichal, R. Mesiar, and E. Pap, Aggregation Functions. Cambridge University Press, Cambridge, 2009.

[6] G. Gudendorf, J. Segers, Extreme-Value copulas. In Proc. of Workshop on Copulas Theory and its Applications. F. Durante et al., eds., 2010

[7] A. Kolesárová, R. Mesiar, and T. Rückschlossová, Power stable aggregation functions. Fuzzy Sets and Systems, doi. 10.1016/j.fss.2013.05.005.

[8] A. Kolesárová, R. Mesiar, 1-Lipschitz power stable aggregation functions. Inform. Sci., submitted.

[9] R.B. Nelsen, An Introduction to Copulas. Vol. 139 of Lecture Notes in Statistics, Springer, New York, second edition, 2006.

[10] A. Sklar, Fonctions de répartition à $n$ dimensions et leurs marges. Publ. Inst. Statist. Univ. Paris 8 (1959) 229-231. 